\title{
POTENTIATION OF SYNEDRELLA NODIFLORA L. FOR INSECTICIDAL ACTIVITY, INSECT REPELLENCY AND BRINE SHRIMP LETHALITY IN THE LABORATORY CONDITIONS
}

\author{
Moniruzzaman, Mohammad Abdullah and Nurul Islam*
}

Department of Zoology, University of Rajshahi, Rajshahi-6205, Bangladesh

*Corresponding Autho, E-mail: n_islamm@yahoo.com; nurulislam@ru.ac.bd

\begin{abstract}
The insecticidal activity, insect repellency and brine shrimp lethality tests of three solvent extracts of Synedrella nodiflora L. were assessed against the adults of Tribolium castaneum (Herbst) under laboratory conditions. The methanol extract found most effective $\left(\mathrm{LD}_{50} 1.229\right.$, 0.823 and $0.569 \mathrm{mg} \mathrm{cm}^{-2}$ for 24,36 and $48 \mathrm{~h}$ of exposure respectively) followed by the chloroform extract $\left(\mathrm{LD}_{50} 3.042,2.773,2.456\right.$ and $2.233 \mathrm{mg}$ $\mathrm{cm}^{-2}$ for $12,24,36$ and $48 \mathrm{~h}$ of exposure respectively) and petroleum ether extract $\left(\operatorname{LD}_{50} 8.416,4.764,3.677,3.392\right.$ and $3.177 \mathrm{mg} \mathrm{cm} \mathrm{cm}^{-2}$ for 12,24 , 36,48 and $60 \mathrm{~h}$ exposure respectively). The $\mathrm{CHCl}_{3}$ and $\mathrm{MeOH}$ extracts showed repellent activity against $T$. castaneum adults at $\mathrm{P}<0.05$ and $\mathrm{P}<0.01$ levels of significance, while the Pet. E. extract did not show any repellency. The similar extracts responded through brine shrimp lethality assay and the Pet. E. extract found most effective $\left(\mathrm{LC}_{50} 321.182,248.151,182.196\right.$ and 140.866ppm) followed by the $\mathrm{MeOH}$ extract $\left(\mathrm{LC}_{50}\right.$ $428.356,72.401,68.397$ and $22.161 \mathrm{ppm}$ for $12,18,24$ and $30 \mathrm{~h}$ of exposure respectively) and the $\mathrm{CHCl}_{3}$ extract $\left(\mathrm{LC}_{50} 669.875,416.605,288.226\right.$ and $248.325 \mathrm{ppm}$ ). The results show the potential of using the $S$. nodiflora extracts for $T$. castaneum suppression.
\end{abstract}

Keywords: Dose-mortality, repellency, Tribolium castaneum, brine shrimp lethality, Artemia salina, Synedrella nodiflora,

সারাংশঃ Synedrella nodiflora L. -এর তিন ধরনের নির্যাসের পোকামাকড় দমন, পোকামাকড় বিতাড়ন ও brine shrimp এর উপর বিষ-কার্যকারিতা যাঁচাইয়ের জন্য চাউলের কেড়িপোকা Tribolium castaneum (Herbst) এর পূর্ণাঙ দশার উপর গবেষণাগার ব্যবস্থায় পরীক্ষা চালান হয়। MeOH নির্যাসটি সবচেয়ে বেশী কার্যকরি পাওয়া যায় (যেখানে ২8, ৩৬, ও 8b- ঘন্টা প্রয়োগকৃত সময়কালের জন্য প্রতি বর্গসেন্টিমিটারে $\mathrm{LD}_{50}$ ছিল যথাক্রমে ১.২২৯, ০.৮-২৩ ও ০.৫৬৯ মি.গ্রা.), এরপর কার্যকরি ছিল $\mathrm{CHCl}_{3}$ निर्याস (যেখানে ১২, ২৪, ৩৬, ও ৪৮ ঘন্টা প্রয়োগকৃত সময়কালের জন্য প্রতি বর্গসেন্টিমিটারে $\mathrm{LD}_{50}$ ছিল যথাক্রমে ৩.০৪২, ২.৭৭৩, ২.৪৫৬ ও ২.২৩৩ মি.গ্রা.); তারপর স্থান পেয়েছে Pet. E. নির্यাসের কার্যকারিতা (যেখানে ১২, ২৪, ৩৬, ৪৮ ও ৬০ ঘন্টা প্রয়োগকৃত সময়কালের জন্য প্রতি বর্গসেন্টিমিটারে LD $_{50}$ ছিল যথাক্রনে ৮.৪১৬, ৪.৭৬৪, ৩.৬৭৭, ৩.৩৯২ ও ৩.১৭৭ মি.গ্রা.)। পূর্ণাঙ T. castaneum এর উপর $\mathrm{CHCl}_{3}$ ও $\mathrm{MeOH}$ নির্যাস বিতাড়ন কার্যকারিতা প্রদর্শন করেছে যথাক্রনে $\mathrm{P}<0.0 ৫$ ও P<০.০১ তাৎপর্যতায়, অবশ্য Pet. E. নির্যাস কোনরূপ কার্যকারিতা দেখায়নি। এই একই নির্যাসগুলি brine shrimp এর বিপরীতে বির্ষক্রিয়া দেখিয়েছে। এক্ষেত্রে Pet. E. নির্যাস ছিল সববেয়ে বেশী কার্यকরি (যেখানে ১২, ১b-, ২৪ ও ৩০ ঘন্টা প্রয়োগকৃত সময়কালের জন্য LC 50 ছিল ৩২১.১b-২, ২৪b-১৫১, ১b-২.১৯৬ ও ১8০.b-৬৬ ppm; এরপর কার্যকরি ছিল $\mathrm{MeOH}$ नির্যাস ( $\mathrm{LC}_{50}$ ছিল 8২৮-৩৫৬, ৭২.৪০১, ৬৮-৩৯৭ ও ২২.১৬১ ppm; সবশেষে $\mathrm{CHCl}_{3}$ नির্যাসের জন্য $\mathrm{LC}_{50}$ ছিল ৬৬৯.৮-৭৫, ৪১৬.৬০৫, ২৮-b.২২৬ ও ২৪b-৩২৫ ppm)।

\section{Introduction}

Synedrelloa nodiflora L. (=Verbesina nodiflora L.) of the family Asteraceae is found in tropical Africa, Asia and West Indies. It is a perennial herb with prostrate stem,often pilose at the nodes with short tricomes to glabrate. Leaves $0.5-1.3 \times 0.5-1.1 \mathrm{~cm}$, broadly ovate to orbiculer, petioles $1-6 \mathrm{~mm}$ long, apex rounded to slightly emerginate, bas rounded to subcordate, globuler puberlent beneath. Flower1-2 in axil, rarely more, up to $5 \mathrm{~mm}$ high, pedicels $2-6 \mathrm{~mm}$ long. Sepals elliptic ovata to elliptic oblong, pubescent, climate corella broadly campanulate, up to $10 \mathrm{~mm}$ wide white. Fruit a globose capsule, $3-4 \mathrm{~mm}$ across often reflex at maturity, seeds $1.5 \mathrm{~mm}$ long, brownis to black subglobose. Flowering and fruiting throughout the year. It is traditionally used in Ghana for the treatment of epilepsy (Woode 2011). In Indonesia the leaves are used as a poultice for sore legs and rheumatism, and juice of leaves used for earache (Rathi and Gopalkrisnan 2005). In Fiji, a decoction of the leaves is used to treat haemorrhoids and diarrhoea. A decoction of the pounded and cooked roots is drunk as a cough- mixture in Africa and in Barbados (Burkill 1985). S. nodiflora were highly biocidal in nature when they were tested on the crop plants like mustard and radish (Ghayal et al. 2008). Patrick et al. (2012) reported the neuropharmacological effects of the $S$. nodiflora extract. The present investigation was carried out to find out the potential of its insecticidal and repellent activity against the red flour beetle, Tribolium castaneum (Herbst), and lethality against the brine shrimp, Artemia salina L. nauplii. The red flour beetle is reddish-brown in color and its antennae end in a three-segmented club (Bousquet 1990). Although small beetles, about $1 / 4$ of an inch long, the adults are longlived and may live for more than three years (Walter 1990), and thus became a suitable lab insect. The Artemia salina belong to a genus of very primordial crustacean (crawfish-crayfish) the Anostraca (Fairy Shrimps). Crawfish of this genus just have a divided exoskeleton made of chitin enhanced protein, no usual crust of chitin (escutcheon) as other crawfish have. There are many species within the genus of Anostraca, but the $A$. salina are very nice to grow, since the rate of successful hatches is very high. 


\section{Materials and Methods}

Collection and preparation of test materials: $S$. nodiflora was collected from the Rajshahi University Campus and identified by Prof. A.T.M. Naderuzzaman and a voucher specimen (No. 48, 10-12-1968) was deposited in the herbarium of the Department of Botany, University of Rajshahi. The plants were chopped into small pieces, dried under shade and powdered using a hand grinder, weighed and placed in separate conical flasks to add Pet. E., $\mathrm{CHCl}_{3}$ and $\mathrm{MeOH}$ (Merck, Germany) $(100 \mathrm{gm} \times 300 \mathrm{ml} \times 2$ times $)$ for $48 \mathrm{~h}$. Filtration was done by Whatman filter paper (made in USA) at $24 \mathrm{~h}$ interval in the same flask followed by evaporation until the extract were left. The extracts was then removed to glass vials and preserved in a refrigerator at $4{ }^{\circ} \mathrm{C}$ with proper labeling.

Collection and culture of test insect: Adults of $T$. castaneum were reared in glass beakers $(500 \mathrm{ml})$ in a standard mixture of whole-wheat flour (ref) with powdered dry yeast $(19: 1)$ in an incubator at $30 \pm 0.5^{\circ} \mathrm{C}$ without light and humidity control for continuous supply of adults during experimentation.

Dose-mortality test: The dose-mortality responses of $S$. nodiflora were observed by surface film method. The concentrations used were $3.57,3.05,2.54,2.04$ and $1.52 \mathrm{mg} \mathrm{cm}^{-2}$ for Pet. E., 3.57, 3.05, 2.54, 2.04, 1.52 and $1.02 \mathrm{mg} \mathrm{cm}^{-2}$ for $\mathrm{CHCl}_{3}$, and 2.54, 2.04, 1.52, 1.02, 0.52 and $0.25 \mathrm{mg} \mathrm{cm}^{-2}$ for $\mathrm{MeOH}$ extract. Each of the doses were diluted in $1 \mathrm{ml}$ of solvent, poured into Petri dishes and allowed to dry. Ten adult beetles were released in each Petri dish, and the experiment of all the doses for each of the extracts were replicated three times. The mortality of the beetles was assessed after 12, 24, 36, 48 and $60 \mathrm{~h}$ of exposures.

Statistical analysis: The mortality (\%) was corrected using Abbott's formula (1925):

$\mathrm{P}_{\mathrm{r}}=\frac{\mathrm{P}_{\mathrm{o}}-\mathrm{P}_{\mathrm{c}}}{100-\mathrm{P}_{\mathrm{c}}} \times 100$

Where, $\mathrm{P}_{\mathrm{r}}=$ Corrected mortality $(\%), \mathrm{P}_{\mathrm{o}}=$ Observed mortality $(\%), \mathrm{P}_{\mathrm{c}}=$ Control mortality $(\%)$. The data were then subjected to probit analysis according to Finney (1947) and Busvine (1971) using a software developed at the University of Newcastle upon Tyne, UK.

Table $1 \mathrm{LD}_{50}$ values of Pet.E., $\mathrm{CHCl}_{3}$ and $\mathrm{MeOH}$ extracts of $S$. nodiflora against $T$. castaneum adults.

\begin{tabular}{lccccc}
\hline \multirow{2}{*}{ Solvent } & \multicolumn{5}{c}{ LD $_{50}$ at different hours } \\
\cline { 2 - 6 } & 12 & 24 & 36 & 48 & 60 \\
\hline Pet. E. & 8.416 & 4.767 & 3.677 & 3.392 & 3.177 \\
$\mathrm{CHCl}_{3}$ & 3.042 & 2.773 & 2.456 & 2.233 & All dead \\
$\mathrm{MeOH}$ & - & 1.229 & 0.523 & 0.569 & All dead \\
\hline
\end{tabular}

Repellent effects: $T$ he $\mathrm{CHCl}_{3}$ and $\mathrm{MeOH}$ extracts of $S$. nodiflora offered a promising repellent effect against $T$. castaneum adults $(\mathrm{P}<0.05$ and $\mathrm{P}<0.01$ respectively) while the Pet. E. extract did not show any repellency (Tables 2 and 3 ).
Repellent activity: The repellency test was adopted from the method (No. 3) of McDonald et al. (1970) with some modifications. Half filter paper discs (Whatman No. 40, diameter $9 \mathrm{~cm}$ ) were treated with the selected doses of $0.079,0.039,0.020,0.010$ and $0.005 \mathrm{mg} \mathrm{cm}^{-2}$ for Pet. E. extract and were then attached lengthwise, edge-to-edge, to a control half-disc with adhesive tape and placed in the Petri dishes. The orientation was changed in the two remaining replicates to avoid the effects of any external directional stimulus affecting the distribution of the test insects. Ten adult insects were released in the middle of each of the filter paper circles. The similar process was done for the $\mathrm{CHCl}_{3}$ and $\mathrm{MeOH}$ extracts respectively.

Each concentration of each solvent was tested for five times. Insects that settled on each of the non-treated half of the filter paper discs were counted after $1 \mathrm{~h}$ and then observed repeatedly at hourly intervals for five hours. The average of the counts was converted to percent repellency $(P R)$ using the formula of Talukder and Howse $(1993,1995): P R=(\mathrm{Nc}-5) \times 20$, where, $\mathrm{Nc}$ is the percentage of insects on the untreated half of the disc.

Brine shrimp nauplii lethality test: Brine shrimp eggs were purchased from Kalabagan, Dhaka and kept in aerated seawater at room $\left(25-30^{\circ} \mathrm{C}\right)$ temperature and took $30-48 \mathrm{~h}$ to give nauplii. The series of concentration were 499.3, 249.6, 124.8, 62.5 and 31.2ppm for Pet. E., 499.5, 249.7, 124.8, 62.5 and 31.5ppm for $\mathrm{CHCl}_{3}$ and 600, 300, 150, 75 and $37.5 \mathrm{ppm}$ for $\mathrm{MeOH}$ extract. Ten freshly hatched nauplii were added to each of the test tubes with different concentrations mentioned earlier and observed mortality after $6,12,18,24$ and $30 \mathrm{~h}$ of exposures. The data was then subjected to probit analysis.

\section{Results and Discussion}

Dose mortality effects: The dose-mortality assay of Pet. E., $\mathrm{CHCl}_{3}$ and $\mathrm{MeOH}$ extracts are represented in Table 1. The $\mathrm{MeOH}$ extract offered highest mortality giving $\mathrm{LD}_{50}$ values ranged between 1.229 to $0.569 \mathrm{mg} \mathrm{cm}$ followed by the $\mathrm{CHCl}_{3}$ extract ranged between 3.042 to $2.233 \mathrm{mg} \mathrm{cm}^{-2}$ and Pet. E. extract ranged between 8.416 to $3.177 \mathrm{mg} \mathrm{cm}^{-2}$. 
Table 2 Percent repulsion values and the arcsin transformed data of the Pet. E., $\mathrm{CHCl}_{3}$ and $\mathrm{MeOH}$ extracts of S. nodiflora gainst $T$. castaneum adults.

\begin{tabular}{|c|c|c|c|c|c|c|}
\hline \multirow[t]{2}{*}{ Type of extract } & \multirow[t]{2}{*}{$\mathrm{cm}^{-2} \mathrm{mg}$} & \multicolumn{5}{|c|}{$\begin{array}{l}\text { Percent repulsion } \mathrm{PR}=(\mathrm{Nc}-5) \times 20 \text { in hours } \\
\quad(\text { Arcsin transformed values for ANOVA) }\end{array}$} \\
\hline & & 1 & 2 & 3 & 4 & 5 \\
\hline \multirow{5}{*}{ Pet.E } & 10 & $\begin{array}{c}40 \\
(39.23)\end{array}$ & $\begin{array}{c}66.6 \\
(54.70)\end{array}$ & $\begin{array}{c}73.2 \\
(58.82)\end{array}$ & $\begin{array}{c}73.2 \\
(58.82)\end{array}$ & $\begin{array}{c}80 \\
(63.43)\end{array}$ \\
\hline & 5 & $\begin{array}{l}100 \\
(90)\end{array}$ & $\begin{array}{l}100 \\
(90)\end{array}$ & $\begin{array}{c}93.2 \\
(74.88)\end{array}$ & $\begin{array}{c}86.6 \\
(68.53)\end{array}$ & $\begin{array}{l}100 \\
(90)\end{array}$ \\
\hline & 2.5 & $\begin{array}{l}100 \\
(90)\end{array}$ & $\begin{array}{c}93.2 \\
(74.88)\end{array}$ & $\begin{array}{c}93.2 \\
(74.88)\end{array}$ & $\begin{array}{c}86.6 \\
(68.53)\end{array}$ & $\begin{array}{c}80 \\
(63.43)\end{array}$ \\
\hline & 1.25 & $\begin{array}{c}86.6 \\
(68.53)\end{array}$ & $\begin{array}{l}100 \\
(90)\end{array}$ & $\begin{array}{c}66.6 \\
(54.70)\end{array}$ & $\begin{array}{c}86.6 \\
(68.53)\end{array}$ & $\begin{array}{c}93.2 \\
(74.88)\end{array}$ \\
\hline & 0.625 & $\begin{array}{c}73.2 \\
(58.82) \\
\end{array}$ & $\begin{array}{c}86.6 \\
(68.53) \\
\end{array}$ & $\begin{array}{c}86.6 \\
(68.53) \\
\end{array}$ & $\begin{array}{c}53.2 \\
(48.83)\end{array}$ & $\begin{array}{c}60 \\
(50.77) \\
\end{array}$ \\
\hline \multirow{4}{*}{$\mathrm{CHCl}_{3}$} & 10 & $\begin{array}{c}93.2 \\
(74.88) \\
80 \\
(63.43)\end{array}$ & $\begin{array}{c}100 \\
(90) \\
73.2 \\
(58.82)\end{array}$ & $\begin{array}{c}100 \\
(90) \\
73.2 \\
(58.82)\end{array}$ & $\begin{array}{c}100 \\
(90) \\
73.2 \\
(58.82)\end{array}$ & $\begin{array}{c}100 \\
(90) \\
73.2 \\
(58.82)\end{array}$ \\
\hline & 2.5 & $\begin{array}{c}60 \\
(50.77)\end{array}$ & $\begin{array}{c}-33.4 \\
(35.30)\end{array}$ & $\begin{array}{c}73.2 \\
(58.82)\end{array}$ & $\begin{array}{c}-13.4 \\
(21.39)\end{array}$ & $\begin{array}{c}-40 \\
(39.23)\end{array}$ \\
\hline & 1.25 & $\begin{array}{c}-6.6 \\
(4.66)\end{array}$ & $\begin{array}{c}6.6 \\
(4.66)\end{array}$ & $\begin{array}{c}-13.2 \\
(21.30)\end{array}$ & $\begin{array}{c}-20 \\
(26.57)\end{array}$ & $\begin{array}{c}-20 \\
(26.57)\end{array}$ \\
\hline & 0.625 & $\begin{array}{c}-26.8 \\
(31.18) \\
\end{array}$ & $\begin{array}{c}-33.4 \\
(35.30) \\
\end{array}$ & $\begin{array}{c}-40 \\
(39.23) \\
\end{array}$ & $\begin{array}{c}-93.2 \\
(74.88) \\
\end{array}$ & $\begin{array}{c}-73.4 \\
(58.95) \\
\end{array}$ \\
\hline \multirow{5}{*}{$\mathrm{MeOH}$} & 10 & $\begin{array}{c}00 \\
(00)\end{array}$ & $\begin{array}{c}00 \\
(00)\end{array}$ & $\begin{array}{c}00 \\
(00)\end{array}$ & $\begin{array}{c}00 \\
(00)\end{array}$ & $\begin{array}{c}00 \\
(00)\end{array}$ \\
\hline & 5 & $\begin{array}{c}-53.2 \\
(46.83)\end{array}$ & $\begin{array}{c}-73.4 \\
(58.82)\end{array}$ & $\begin{array}{c}-53.2 \\
(46.83)\end{array}$ & $\begin{array}{c}-53.2 \\
(46.83)\end{array}$ & $\begin{array}{c}-53.2 \\
(46.83)\end{array}$ \\
\hline & 2.5 & $\begin{array}{c}-20 \\
(26.57)\end{array}$ & $\begin{array}{c}-26.8 \\
(31.18)\end{array}$ & $\begin{array}{c}-33.4 \\
(35.30)\end{array}$ & $\begin{array}{c}-33.4 \\
(35.30)\end{array}$ & $\begin{array}{c}-20 \\
(26.57)\end{array}$ \\
\hline & 1.25 & $\begin{array}{c}-53.2 \\
(46.83)\end{array}$ & $\begin{array}{c}-46.8 \\
(43.17)\end{array}$ & $\begin{array}{c}26.6 \\
(31.05)\end{array}$ & $\begin{array}{c}26.6 \\
(31.05)\end{array}$ & $\begin{array}{c}00 \\
(00)\end{array}$ \\
\hline & 0.625 & $\begin{array}{c}-53.2 \\
(46.83)\end{array}$ & $\begin{array}{c}-46.8 \\
(43.17)\end{array}$ & $\begin{array}{c}-73.4 \\
(58.95)\end{array}$ & $\begin{array}{c}-73.4 \\
(58.95)\end{array}$ & $\begin{array}{c}-60 \\
(50.77)\end{array}$ \\
\hline
\end{tabular}

Table 3 Repellency effect of the Pet. E., $\mathrm{CHCl}_{3}$ and $\mathrm{MeOH}$ extracts of $\mathrm{S}$. nodiflora against $T$. castaneum adults.

\begin{tabular}{ccc}
\hline \multirow{2}{*}{ Solvent } & Between doses $(\mathrm{df}=4)$ & Between time interval $(\mathrm{df}=4)$ \\
\cline { 2 - 3 } & F-value & F-value \\
\hline Pet. E. & 1.043 & 5.95 \\
$\mathrm{CHCl}_{3}$ & 0.883 & $22.29^{*}$ \\
$\mathrm{MeOH}$ & 1.083 & $24.85^{* *}$
\end{tabular}

Brine shrimp lethality effect: The brine shrimp lethality for Pet. E., $\mathrm{CHCl}_{3}$ and $\mathrm{MeOH}$ extracts of $S$. nodiflora represented in Table 4. The Pet. E. extract offered the highest activity with $\mathrm{LC}_{50}$ values 321.182 to $140.866 \mathrm{ppm}$, followed by the $\mathrm{MeOH}$ extract with $\mathrm{LC}_{50}$ values 428.356 to $22.161 \mathrm{ppm}$ and for the $\mathrm{CHCl}_{3}$ extract with $\mathrm{LC}_{50}$ values 669.875 to $248.325 \mathrm{ppm}$ all for 12,18 , 24 and $30 \mathrm{~h}$ of exposures respectively.
Table $4 \mathrm{LC}_{50}$ values of Pet. E., $\mathrm{CHCl}_{3}$ and $\mathrm{MeOH}$ extracts of $S$. nodiflora against $A$. salina nauplii.

\begin{tabular}{ccccc}
\hline \multirow{2}{*}{ Solvent } & \multicolumn{4}{c}{ Duration of exposure in hours } \\
\cline { 2 - 5 } & 12 & 18 & 24 & 30 \\
\hline Pet.E & 321.182 & 248.151 & 182.196 & 140.866 \\
$\mathrm{CHCl}_{3}$ & 669.875 & 416.605 & 288.226 & 248.325 \\
$\mathrm{MeOH}$ & 428.356 & 72.461 & 68.397 & 22.161 \\
\hline
\end{tabular}


These findings receive supports from previous researchers' achievements. The whole plant extract of the $S$. nodiflora has shown antioxidant and cytotoxic activity (Dutta et al. 2012). Insecticidal activity of aerial parts of S. nodiflora on Spodoptera latura has been reported by Rathi and Gopalkrishnan (2005). Extracts of S. nodiflora (L). Gaertn. displayed anti-inflammatory, analgesic, antinociceptive, antipyretic and insecticidal properties (Abad et al. 1996; Forestieri et al. 1996; Rathi and Gopalakrishnan 2006; Woode et al. 2009). Bhogaonkar (2011) mentioned that S. nodiflora (L.) Gaertn. is a less known medicinal plant and it has good antibacterial and antifungal activity. Traditionally, the leaves are used as poultice for rheumatism and the juice of the leaves is used for earache in India (Rathi and Gopalakrishnan 2006). The ethanol extract of S.nodiflora has antioxidant and antibacterial properties on account of phenolic compounds of flavonoids (Sumi 2011). The hydro-ethanolic extract of $S$. nodiflora exhibits anxiogenic-like effects which could be attributed to sedation and reduction in locomotor activity (Woode et al. 2011). Thus, a comprehensive phytochemical analysis of the plant for its insecticidal and repellent components, as well as the physiological studies of the active ingredients are very much to be solicited for their effective use in the future pest control and pharmaceutical endeavors.

Acknoweledgement : The authors are grateful to the University Grants Commission of Bangladesh and Rajshahi University for a university research grant. They would like to thank the Chairman, Department of Zoology for providing laboratory facilities.

\section{References}

Abad MJ, Bermejo P, Carretero E, Martinez-acitores C, Noguero B, Villar A. 1996. Antiinflammatory activity of some medicinal plant extracts from Venezuela. J. Ethnopharmacol. 55: 63-68.

Abbott WS. 1925. A method of computing the effectiveness of an insecticide, J. Econ. Entomol. 18, 265-267.

Bhogaonkar PY, Dagawal MJ, Ghorpad DS. 2011. A pharmacological studies and antimicrobial activity of Synedrella nodiflora L. J. Bioscience Discover. 3: 317-321.

Bousquet MF, Klocke JA, Wurtele ES, and Bollinger WH. 1990. Natural plant chemicals: Sources of industrial and medicinal materials. Science. 228: 1154-1160.

Burkill HM. 1985. The useful plants of West Tropical Africa, Vol. 1 NHBS Ltd. Kew England.

Busvine JR. 1971. A critical review of the techniques for testing insecticides. Commonwealth Agricultural Bureau, London, 345 pp.
Dutta M, Nath AK, Uddin MZ, Hossain MA, Morshed MM, Kawser HM. 2012. In vitro antioxidant, total phenolic content and Brine shrimp lethality studies of Synedrella nodiflora L. International $J$. Pharmaceutic. Sci. and Res. 3(5): 1528-1531.

Finney DJ. 1947. Probit analysis: a statistical treatment of the sigmoid response curve. Campridge University Press, London. 333 pp.

Forestieri AM, Monforte MT, Ragusa S, Trovato A, Iauk L. 1996. Antiinflammatory, analgesic and antipyretic activity in rodents of plant extracts used in African medicine. Phytotherapy Research 10: 100-106.

Ghayal NA, Dhumal KN, Deshpande NR, Shah SM and Ruikar AD. 2008. Studies on allelochemicals in Synedrella nodiflora and impact of its leaf leachates on germination and seedling growth of Radish (Raphanus sativus) and Mustard (Brassica juncea). Asian J. Chem. 20(8): 6114 - 6120.

Idu M, Onyibe HI. 2007. Medicinal plants of Edo state Nigeria. J. Medicinal Plant Res. 2: 32-41.

McDonald LL, Guy RH, Speirs RD. 1970. Preliminary evaluation of new candidate materials as toxicants, repellents and attractants against stored-product insects. Marketing Research Report No. 882. Agricultural Research Service, US Department of Agriculture, Washington, DC.

Patrick A, Eric W, Samuel BK. 2012. Anticonvulsant and related neuropharmacological effects of the whole plant extract of Synedrella nodiflora (L.) Gaertn (Asteraceae). J. Pharm. \& Bio Allied Sci. 4: 140-148.

Rathi MJ, Gopalakrishnan S. 2005. Insecticidal activity of aerial on Spodoptera litura. J. Central European Agric. 6: 223-228.

Rathi MJ, and Gopalakrishnan S. 2006. Insecticidal activity of aerial parts of Synedrella nodiflora Gaertn. (Compositae) on Spodoptera litura (Fab.).Central European Agric. 7(2): 289-296.

Suleiman MM, Dzenda T, Sani CA. 2008. Antidiarrhoeal activity of the methanol stem-bark extract of Annona senegalensis Pers. (Annonaceae). J. Ethnopharmacol. 116: 125-130.

Sumi, W, Kang, J, Teng, K, Hwei, L. 2011. Antibacterial and Antioxidant Activities of Synedrella nodiflora (L.) Gaertn (Asteraceae). J. Complementary and Integrative Medicine: 8(1): Article 13.

Talukder FA, Howse PE. 1993. Deterrent and insecticidal effects of extracts of pithraj, Aphanamixis polystachya (Mcliaceae) against Tribolium castaneum in storage. J. Chem. Ecol. 19: 2463-2471.

Talukder FA, Howse PE. 1995. Evaluation of Aphanamixis polystachya as a source of repellents, antifeedants, toxicants and protectants in storage against Tribolium castaneum (Herbs). J. Stored Prod. Res. 31: 55-61.

Walter VE. 1990. Stored product pest. Franzak and Foster Co., Cleveland, OH. pp. 526-529.

Woode E, Amoateng P, Ansah C, Duwiejua M. 2009. Antinociceptive effects of an ethanolic extract of the whole plant of Synedrella nodiflora (L.) Gaertn in mice: Involvement of adenosinergic mechanism. J. Pharmacol. and Toxicol. 4: 17-29.

Woode E, Amoateng P, Abotsi WKM. 2011. Ethopharmacological analysis of the effects of the whole plant extract of Synedrella nodiflora (L.) Gaertn (Asteraceae) in murine models. Der Pharmacia Sinica, 2(2): 54-67.

Zahan R, Nahar L, Haque A, Mosaddik A, Fazal A, Alam Z, Haque EM. 2012. Antidiarrhoeal and hypoglycemic effects of Synedrella nodiflora. Phytopharmacol. 2(2): 257-264.

Manuscript received on 5 September 2012 and revised on 29 December 2012 